\title{
LA CROISADE CONTRE LE MAUVAIS CINÉMA \\ Sur les préambules et le démarrage du contrôle belge des films (1912-1929)
}

\author{
Liesbet Depauw $^{1}$ et Daniel Biltereyst ${ }^{2}$
}

\section{Introduction}

Dans la littérature internationale concernant la censure des films, la Belgique occupe une place particulière.Tout comme dans plusieurs pays occidentaux, le parlement belge a entériné après la Première Guerre mondiale une législation qui devait mettre fin à ce qui était souvent indiqué par "la question du film". Mais la loi du 1er septembre 1920, généralement nommée la Loi Vandervelde, présentait de subtiles différences par rapport aux législations étrangères courantes en matière de censure de films. La Belgique ne connaissait pas de censure obligatoire, mais instaura un contrôle des films librement consenti pour les projections de films pour les enfants de moins de 16 ans. Le royaume ne connaissait pas de censure (de films) obligatoire pour les adultes, si bien

1 Daniel Biltereyst est professeur de cinéma et d'études culturelles des médias au Département des Sciences de la Communication, Université de Gand (UGent).

2 Liesbet Depauw est chercheur près du Fonds de Recherche Scientifique (FWOVlaanderen) et travaille au Département des Sciences de la Communication, Université de Gand (UGent).

Recherches en communication, $\mathrm{n}^{\circ} 24$ (2005). 
que les distributeurs et les producteurs de films avaient toute liberté de proposer leurs films directement aux exploitants de salles de cinéma. Seulement dans le cas où ils visaient un public plus jeune, il leur fallait l'approbation de l'instance officielle de contrôle des films, qui entama ses activités début 1921.

Cette législation libérale (en apparence) allait conduire au fait qu'en Belgique le contrôle central des autorités sur l'offre dans les salles était moindre que celui qui prévalait dans de nombreux pays occidentaux. Ainsi, plusieurs films controversés et interdits à l'étranger purent être projetés en Belgique. D'autre part, la Loi Vandervelde était généralement considérée par le secteur du film comme une législation de censure camouflée. Comme les enfants et les ménages constituaient une cible importante de l'industrie cinématographique, des raisons commerciales contraignaient pour ainsi dire les distributeurs et les producteurs de présenter leurs films à la commission de contrôle, dont l'approche sévère devint rapidement la cible d'attaques virulentes du secteur.

Contrairement à la recherche dans la plupart des pays voisins, l'histoire du contrôle officiel belge des films est un terrain en friche. Le présent article s'engage dans le débat public et dans les initiatives législatives au moment des préambules et de la mise en route de la commission officielle du contrôle belge des films. A cet effet, nous retournons à la période précédant immédiatement la Première Guerre mondiale, lorsque la question du film éclata dans toute sa violence, surtout au niveau des communes. Sur base de recherches en cours concernant les pratiques de contrôle, nous approfondirons ensuite le démarrage et la portée du contrôle des films jusqu'à la fin des années 1920.

\section{La croisade contre le mauvais film s'accélère (1912-1914)}

Bien que la Belgique n'avait pour ainsi dire pas de production cinématographique, le pays était caractérisé par une activité intense de distribution et d'exploitation. Avant la Première Guerre mondiale, Bruxelles était considéré comme un centre important de distribution cinématographique, tandis que la ville avait un grand nombre de cinémas. La popularité énorme du film conduisit en Belgique, comme dans d'autres pays, à un débat intense au sujet du cinéma comme un danger menaçant les enfants. Des avocats, des juges de la jeunesse, des groupes de pression à caractère moral et des politiciens firent des années durant diligence contre l'influence présumée nocive du cinéma, principalement pour les enfants. Au cours de cette période, le film était 
souvent considéré comme une école perfide de criminalité, d'immoralité et de violence ${ }^{1}$.

Le débat belge sur l'influence du "mauvais cinéma" remonte à la période avant la Première Guerre mondiale, mais s'est accéléré en 1912. Le débat était concentré sur trois grands thèmes : le contenu des films, les circonstances dans lesquelles les films sont projetés et l'attirance destructive du medium.

Généralement, la crainte que les enfants subissent une influence négative émanant des images immorales se trouvait au coeur du débat. Outre des images d'adultère, de désobéissance et de débauche - qui érodaient les valeurs et les normes catholiques - , la crainte qu'il pouvait y avoir un lien de cause à effet entre la délinquance juvénile et les scènes criminelles des films était bien vivante. Plusieurs juges de la jeunesse, parmi lesquels Paul Wets, Prosper Peiren et Albert Soenens, accusèrent à plusieurs reprises le cinéma d'être une cause importante de comportements criminels de la jeunesse. Ainsi, le Bruxellois Paul Wets, un juge de la jeunesse très influent, avança que :

Le cinéma est sans conteste le pourvoyeur par excellence des juridictions des enfants. Au cours des centaines d'interrogatoires auxquels il nous fut donné de procéder, il n'y a pas $5 \%$ d'enfants qui n'avouent pas spontanément, qu'ils sont spectateurs assidus des théâtres cinématographiques².

Dans la Chambre, un discours similaire fut repris. Ainsi le parlementaire chrétien-démocrate Rombouts était d'avis que le cinéma avait "souvent directement enseigné le crime" aux enfants 3 .

La concentration sur le contenu des films se manifestait aussi dans l'inquiétude à propos des titres de films et des affiches dans la rue. Un bref regard sur les titres de films de cette époque met cette crainte en perspective. Ainsi on vit apparaître entre 1910 et 1921 des titres comme LES TROIS AMANTS, BIÈRE QUI ROULE, LE BON PETIT DIABLE, MARIAGE SECRET, LA PEUR DE MARIAGE et LE ROMAN D'UNE

1 Dans le débat belge concernant la question du film des notions telles que "l'école du vice", "l'école de crime" ou "l'école de l'immoralité et de la perversion" reviennent constamment, comme en France d'ailleurs. Voir P. Wets, Une Enquête sur les Spectacles Cinématographiques à Bruxelles, Bruxelles, 1920, p. 6.

2 P. Wets, La guerre et l'enfant, Mol, 1919, p. 33.

3 Chambre des Députés - Actes parlementaires, 5 mars 1920, p. 777. 
PROSTITUÉE, qui ne promettaient pas grand-chose de bon d'après les critiques $^{1}$.

La conviction que le cinéma était nuisible pour la jeunesse, allait cependant au delà de son seul contenu. Le fait que les enfants aillent tout seuls au cinéma et qu'ils puissent y rencontrer des jeunes de l'autre sexe dans la pénombre était mentionné dans presque chaque rapport concernant les conséquences nuisibles du cinéma. Les conditions souvent peu hygiéniques de la plupart des salles de cinéma se trouvaient également dans la ligne de mire. D'après les auteurs, les enfants ont besoin de lumière et d'oxygène. Le séjour prolongé dans l'espace obscur mettrait la santé et surtout les yeux des enfants en danger. En outre, les bobines de films risquaient de s'enflammer, ce qui pouvait donner lieu à la panique ${ }^{2}$.

Une dernière crainte provenait de l'attirance présumée qu'exerçait le cinéma sur les jeunes. Les partisans d'une réglementation en matière de cinéma étaient d'avis que les films pouvaient constituer une obsession pour les enfants. Plusieurs rapports et écrits contre le cinéma mentionnaient donc que les enfants volaient parfois de l'argent pour pouvoir s'acheter des billets d'entrée. En raison de cette obsession, d'autres activités "saines" telles que le sport et la lecture seraient compromises et les études reléguées au second plan $^{3}$. La croisade contre le cinéma ne se concentra pas seulement sur le contenu des films joués, mais présenta le danger comme étant une donnée plus vaste, sociale. Pourtant la loi de 1920 allait se préoccuper uniquement du contrôle du contenu des films.

Ces objections contre le cinéma s'intensifièrent en 1912, lorsque des mesures furent prises, essentiellement au niveau des communes. $\mathrm{Au}$ mois de juin de cette année-là, une action publique de protestation eut lieu à Ixelles à l'encontre du film français ZIGOMAR, ROI DES VOLEURS (1911, Victorin Jasset) ${ }^{4}$. Les protestations étaient si violentes

1 Ces titres renvoient aux films suivants: LOVERS THREE (1913, 1913, EU); BEER MUST GO DOWN (1916, Eddy Lyons \& Lee Moran, EU); A GOOD LITTLE DEVIL (1914, Edwin S. Porter \& S. Dawley, EU); SECRET MARRIAGE (1918, T. Ricketts, EU); A FUGITIVE FROM MATRIMONY (1919, Henry King, EU); EN GATEPIGES ROMAN (1912, Adam Eriksen, Norvège).

2 V. Plas, L'enfant et le cinéma, Bruxelles, 1914, p. 7.

3 E. Beco, La croisade entreprise contre le mauvais cinéma pendant la guerre, Turnhout, 1919.

4 R. de Ryckère, Revue critique - Lettre de Belgique-Les cinémas et les romans policiers, in: Archives d'Anthropologie Criminelle de Médecine Légale et de 
que le conseil communal d'Ixelles délibéra longuement le 25 juin 1912 sur les moyens d'éviter de telles actions à l'avenir. La question fondamentale, qui était aussi à l'ordre du jour dans d'autres communes, se rapportait à la compétence des communes en matière de réglementation du cinéma, en particulier au sujet du contenu qualifié d'immoral. La commune décida de remettre provisoirement la question et de la traiter ultérieurement au cours d'une assemblée générale des bourgmestres de Bruxelles ${ }^{1}$.

$\mathrm{Au}$ cours de leur assemblée générale, nommée Collège des Bourgmestres, les bourgmestres de Bruxelles parvinrent rapidement à un point de vue unanime: les communes et les villes n'avaient aucune compétence au niveau de la surveillance et de la sauvegarde de la moralité. D'après les bourgmestres, l'article 97 de la loi communale énonçait clairement que le conseil communal et le collège des échevins avaient pour mission de surveiller le calme et l'ordre public, mais la loi sur les communes n'allait pas au delà. Les bourgmestres interprétèrent l'ordre public comme quelque chose de matériel et parvinrent donc à la conclusion que la surveillance de l'ordre moral n'était pas réglée par la loi. Ils reconnurent pourtant de façon explicite le problème du film immoral, essayant d'inciter dans leur déclaration en commun à la prise d'une initiative législative nationale ${ }^{2}$. Malgré ce point de vue commun ferme, les critiques accusèrent les bourgmestres dans le débat public de laissez aller. Tandis que des journaux tels que Le Soir donnèrent encore dans une certaine mesure une image nuancée et placèrent souvent deux opinions opposées d'experts côte à côte, les critiques furent très clairs. Ainsi, le représentant socialiste Vinck avança le 14 mai 1914 au Parlement :

Je tiens néanmoins à faire une observation au sujet de la répression des spectacles cinématographiques. Si l'on y met un terme, nous aurons à déplorer, d'ici peu de temps, des ravages dans la mentalité de la jeunesse de notre pays. Ne venez pas dire, monsieur le ministre, que vous n'avez pas encore pu constater si les bourgmestres ne font pas leur devoir. Nous pouvons dire maintenant, qu'ils ne le font pas ${ }^{3}$.

Psychologie Normale et Pathologique, 27, 1912, pp. 941-945.

Bulletin Communal d'Ixelles, séance du 25 juin 1912, pp. 554-572.

Bulletin Communal de la Ville de Bruxelles, 3 février 1913, pp. 83-94.

3 Sénat-Actes parlementaires, 19 mai 1914, p. 783. 
Vital Plas, un critique éminent dans le débat, tint un plaidoyer fort pour une interprétation extensive de la compétence des villes et communes, où le bourgmestre devrait occuper une place importante. Plas étaya sa thèse au moyen d'une vaste étude sur l'influence du film sur la jeunesse. A l'initiative de la Société Belge de Pédotechnie, Plas collabora à partir de 1912 à une recherche internationale d'inventorisation au sujet des mesures prises au niveau communal en matière de censure du film. Cette étude, publiée début 1914, mit déjà en lumière la diversité des mesures prises dans plusieurs pays. Dans son exposé final, Plas déplora que la Belgique fut en reste. Seul un nombre limité de villes et de communes avait pris des mesures pour chasser les images immorales de l'écran ou pour interdire l'accès des salles de cinéma aux enfants non accompagnés.

Sur base de l'enquête de Plas, plusieurs recommandations furent formulées pour parvenir à un système uniformisé. D'après le rapport, une commission indépendante aurait dû être créée, qui contrôlerait tous les films pour envoyer ensuite cette information aux communes. Il est à remarquer que Plas insistait toujours sur le fait que la réglementation du film faisait partie de la mission des bourgmestres. Les conclusions de Plas offraient déjà une ébauche pouvant servir de base à la loi de 1920.

\section{La Première Guerre mondiale et le mouvement anticinéma- tographique}

L'invasion des Allemands et l'occupation de quatre ans qui en a découlé, a contraint de nombreux politiciens, magistrats, juges, prêtres et professeurs à céder leurs compétences en tout ou en partie à l'occupant. Cela ne les retint cependant pas d'entreprendre une croisade très active contre le "mauvais film". Avant la Première Guerre mondiale, la Belgique avait de nombreux groupes de pression comme la Société Belge de Pédotechnie déjà mentionnée et la Ligue contre la licence des étalages et de l'immoralité, qui formulaient des recommandations claires pour éloigner le danger du cinéma de la jeunesse ${ }^{1}$.

Pendant la Première Guerre mondiale, le mouvement "anticinématographique" (nommé par Wets) devint plus fort et fut dirigé de façon plus stricte. L'association la plus importante était le Groupement d'études des leçons de la guerre, fondé par des ténors politiques faisant

1 A. Collette, Moralité et immoralité du cinéma, Liège, 1993. 
autorité tels qu'Emile Beco, gouverneur du Brabant (président), et l'avocat, ultérieurement premier ministre libéral, Paul-Emile Janson (secrétaire). Le Groupement ne considérait pas le cinéma comme entièrement négatif, mais soulignait le potentiel éducatif du médium. Pendant quatre ans, l'association milita en faveur d'une réglementation au niveau communal.

Au sein de l'organisation, sous la présidence de Soenens, conseiller à la Cour de Cassation, un groupe de travail spécial fut fondé, qui se spécialisa dans la question juridique de la répartition des compétences. Dans ses conclusions finales, la commission fit remarquer le caractère relatif de la notion "d'ordre public" qui comprenait d'après elle tout autant les bonnes moeurs et pouvait constituer une légitimation pour une ingérence communale (de la police) dans les séances de cinéma. Le groupe de travail continua cependant à militer pour une législation nationale concluante qui fixerait clairement la compétence des communes en matière de film. Outre l'intervention des autorités locales, l'association fit remarquer l'importance d'une campagne de propagande à grande échelle en collaboration avec les initiatives privées déjà existantes. Comme dernier de cette série des "grands moyens de lutte", l'association plaida aussi en faveur d'impositions fiscales supplémentaires pour l'Etat, les provinces et les villes ${ }^{1}$.

\section{Hanrez, la Loi Vandervelde et le caractère constitutionnel du contrôle des films $(1919$ - 1920)}

Après l'armistice, les travaux du mouvement anticinématographique furent arrêtés. Mais les membres du groupement demeuraient très concernés par la question du film et leurs opinions se retrouvaient régulièrement dans des journaux régionaux et nationaux. L'année 1919 constitua un tournant dans la lutte pour une réglementation plus sévère du film. Les journaux belges publièrent cette année-là régulièrement des articles et des éditoriaux, clouant l'influence néfaste du film au pilori. Cela alla de pair avec la pression pour régler la question du film au niveau national.

En juillet 1919, la première proposition de loi fut déposée au Sénat par le libéral Prosper Hanrez, lui-même membre du Groupement d'études des leçons de la guerre, ainsi que par quelques autres politiciens

1 Voir P. Wets, La guerre et l'enfant, Mol, 1919, pp. 42-46. 
de fractions politiques divergentes. Dans sa brève proposition de loi, Hanrez prévoyait la constitution d'un "comité de surveillance" national, qui devrait contrôler au préalable les "films de cinéma"présentés dans les "salles de théâtre" ". Dans la motivation accompagnant sa proposition de censure de films, Hanrez référa largement à l'influence néfaste du film, avançant de façon lapidaire que "le mal est général, il faut y mettre fin par une mesure générale" (p. 3). La proposition de Hanrez fut finalement rejetée par une commission parlementaire, dans laquelle on fit principalement remarquer le caractère anticonstitutionnel de cette "loi de censure'. En raison de la chute du gouvernement unioniste Delacroix I (catholique-libéral-socialiste) et la dissolution du parlement en novembre 1919, la proposition de Hanrez fut quelque peu remise à plus tard, mais l'idée de censure ou de contrôle des films était lancée.

En mars 1920, pendant le gouvernement unioniste suivant sous la direction du premier ministre catholique Léon Delacroix, la question fut remise sur le tapis. Le ministre de le Justice de l'époque, Emile Vandervelde, un personnage de premier plan du mouvement socialiste international, introduisit avec Jules Destrée, ministre des Sciences et des Arts et appartenant au même parti, un projet de loi qui allait moins loin que celui de Hanrez, et ce à plusieurs niveaux. Ce projet allait donner lieu finalement à la Loi Vandervelde, d'ailleurs toujours en vigueur aujourd'hui.

Le 25 mars 1920 effectivement, la Chambre des Députés trancha. Avec 88 voix contre 44, le projet de loi interdisant l'accès aux salles de cinéma aux enfants de moins de seize ans fut adopté. Un peu moins de deux mois plus tard, le 15 juin 1920, le Sénat adopta lui aussi le projet $^{2}$.

Une raison importante pour laquelle la proposition de Hanrez fut rejetée tandis que celle de Vandervelde et Destrée fut adoptée se rapportait à la base constitutionnelle d'une loi qui pourrait éventuellement instaurer la censure. A ce niveau, il fut régulièrement référé, aussi à l'extérieur des cénacles politiques et en particulier par le secteur du cinéma lui-même, à la protection constitutionnelle de la liberté de la presse en Belgique. Effectivement, lors de l'indépendance en 1830, la Belgique rédigea une constitution très progressiste, libérale, mettant

1 Proposition de loi établissant le contrôle des films cinématographiques, in: Sénat - Actes parlementaires, sessions 1918-1919, 8 juillet 1919 (pièce 109).

2 Chambre des Députés - Actes parlementaires, 25 mars 1920, p. 777. 
expressément l'accent sur la liberté de la presse écrite, la censure étant exclue à jamais.

Vandervelde intégra à sa proposition plusieurs éléments devant éloigner le soupçon de censure des films. Ainsi, il fixa clairement que le contrôle n'était d'aucune façon obligatoire et que les films qui ne passaient pas par la commission de contrôle, pouvaient être joués dans notre pays - sans être accessibles toutefois aux jeunes de moins de 16 ans.

Bien qu'explicitement la Loi Vandervelde ne désirât pas instaurer de censure, les lois économiques de l'entreprise cinématographique et surtout le contrôle sévère jetèrent une ombre sur la législation cinématographique relativement libérale (dans son essence). Bien que le distributeur ne fût légalement pas tenu de présenter ses films, il y était tenu dans la pratique pour des raisons économiques. Le label "ENA" fut vite considéré comme étant néfaste au niveau commercial et fit craindre à l'exploitant une perte de revenus. Cela explique la campagne extrêmement acerbe que le secteur entreprit à l'encontre de la commission de contrôle des films jusque bien avant dans les années 1920.

Les lois économiques au sein du secteur cinématographique octroyèrent ainsi une position de force aux contrôleurs belges des films. Notre étude fait apparaître que les membres de la commission demandèrent de plus en plus fréquemment des coupures afin d'accorder le label désiré "Enfants Admis" au film contrôlé. Ce qui était considéré en théorie comme une mesure clémente, n'ayant aucun rapport avec la censure, allait conduire dans la pratique à la mutilation de centaines de films.

\section{Protestations du secteur cinématographique et la mise en route difficile du contrôle des films $(1920$ - 1922)}

Pendant huit ans, toutes sortes de groupements, de juges de la jeunesse et de politiciens luttèrent pour une loi qui fut finalement promulguée le $1^{\text {er }}$ septembre 1920 . Bien que des voix de protestation s'élevèrent sans ambiguïté jusqu' au sein du parlement et dans la presse, surtout du côté libéral, le secteur cinématographique lui-même était de toute évidence très opposé à la loi du contrôle des films. Les réactions étaient si violentes que cela allait encore durer jusqu'au mois de mai 1922 avant que la commission de contrôle pût effectivement se mettre à l'œuvre au moyen d'un Arrêté Royal. Une période de transition de plus 
d'une année fut requise avant que la commission pût déployer pleinement ses activités normales ${ }^{1}$.

Outre par un marché florissant de cinémas, le secteur cinématographique belge était caractérisé au début des années 1920 par un secteur de distribution très éparpillé et vivant. Ainsi la Belgique comptait à cette époque plus de 50 distributeurs ayant souvent leurs bureaux près de la gare du nord de Bruxelles. Le secteur de distribution et d'exploitation comptait de nombreux groupes de pression, parmi lesquels la Chambre Syndicale Belge de Cinématographie, la Fédération Belge Cinématographique et la Ligue Nationale du Cinéma ${ }^{2}$. Ces associations se réunirent en 1920 et formèrent temporairement la Ligue Nationale Belge pour la Défense des Intérêts du Cinématographe, de toute évidence avec pour objectif de combattre la loi sur le cinéma.

Au cours de 1920 et de 1921, les membres de la ligue envoyèrent régulièrement des délégations aux politiciens, parmi lesquels Vandervelde et Destrée en personne, tandis qu'ils publièrent aussi continuellement des lettres ouvertes et adressèrent une lettre de doléances au roi. En outre, ils organisèrent une "enquête auprès du peuple", projetèrent dans leurs salles de petits films de propagande contre le contrôle des films et annoncèrent en mars 1921 une grève générale, qui ne fut que partiellement suivie. Finalement la Ligue décida le 13 avril 1921 de boycotter la loi en ne présentant aucun film à la commission de contrôle des films ${ }^{3}$.

Les distributeurs et exploitants s'opposèrent à partir d'intérêts différents. Les distributeurs craignirent qu'une trop grande partie de leurs films serait refusée par la commission de contrôle des films. Les films refusés signifiaient effectivement des coûts supplémentaires élevés pour un distributeur. Outre les frais de contrôle en soi (montant à payer au prorata de la longueur du film contrôlé), un film portant le label "ENA" trouvait plus difficilement la route des salles de cinéma. D'après un rapport de la Fédération Belge Cinématographique au sujet des plaintes des "cinématographistes" belges de mars 1921, un exploitant de salle perdrait au moins soixante-deux pour cent de son public si les enfants n'étaient plus admis194. Les exploitants de salles étaient

1 L'Arrêté Royal du 11 mai 1922 a été publié au Moniteur Belge, 92e année, 22 \& 23 mai 1922, pp. 3877-8780.

2 Voir e.a. A. Collette, Moralité et immoralité du cinéma, Liège, 1993.

La question des cinémas, Demain, 31 mars 1921, p. 3.

4 Voir Rapport sur les Griefs de la Cinématographie Belge : La Grande Réunion du 
aussi convaincus du fait que la nouvelle loi allait créer un manque de films pour toute la famille.

Les protestations massives du secteur cinématographique firent que Vandervelde promit, afin d'éviter une grève générale, d'instaurer par Arrêté Royal une mesure de transition à partir du 26 mai 1921. Cette mesure fixait que les distributeurs pouvaient faire contrôler pendant quelques mois leurs films à titre gracieux et ceci sur base uniquement du scénario.

Fin mai 1921, le ministre de la Justice fit parvenir une circulaire importante aux contrôleurs, dans laquelle Vandervelde répétait encore une fois que sa loi "n'était pas une loi de censure", n'ayant "pas à se préoccuper de la tendance politique, philosophique ou religieuse des films ${ }^{1}$ ". Fin décembre 1920, lors de l'installation de la commission à la Montagne aux Herbes Potagères à Bruxelles, Vandervelde avait déjà formulé ses objectifs de façon très explicite à l'égard de la presse ${ }^{2}$, mais dans cette nouvelle circulaire il soulignait une fois de plus que la loi ne s'ingérait nullement dans la projection de films pour les personnes majeures. Son objectif était de protéger la jeunesse ${ }^{3}$. Dans cette circulaire, il mentionnait explicitement ce qui retiendrait l'attention des nouveaux contrôleurs, notamment les films montrant des actions criminelles, ainsi que les films s'appuyant sur des actes de cruauté et de violence ${ }^{4}$.

\section{Le contrôle des films découvre les ciseaux (1921 - 1929)}

Il n'est guère facile de répondre à la question de savoir dans quelle mesure la commission de contrôle des films se tint à ces directives. Mais il est certain que les membres de la commission de contrôle des films, présidée de 1921 à 1928 par le juge des enfants Gombault, se mirent à contrôler de plus en plus sévèrement dans les années 1920. Une analyse systématique de leurs pratiques de contrôle, en particulier les descrip-

Mardi 15 mars, in Revue Belge du Cinéma, 20 mars 1921, p. 36.

1 "Voorschriften aan de Afgevaardigden", Bruxelles 24 mai 1921, RAB (Rijksarchief Beveren), EA DEAD 2001, pièce 1172.

2 L'Epuration du cinéma. Installation de la Commission de Contrôle, in Le Soir, 23 décembre 1920.

3 "Voorschriften aan de Afgevaardigden", Bruxelles 24 mai 1921, RAB (Rijksarchief Beveren), EA DEAD 2001, pièce 1172, p. 1.

4 Ib., p. 3. 
tions des coupures, fait apparaitre qu'ils demandèrent en tout cas de plus en plus de coupures avant d'autoriser le film. Tandis qu'en 1923 les coupures ne s'appliquèrent qu'à trois pour cent des films présentés, ce pourcentage s'élevait à la fin des années 1920 à trente pour cent. Les descriptions des coupures montrent les sensibilités de la commission, qui dans un contexte plus large pourraient sans doute constituer un indicatif des points névralgiques de la société.

Ainsi, les contrôleurs étaient surtout heurtés par la représentation de la violence et de la criminalité. Dans un peu moins de $70 \%$ de toutes les coupures réclamées entre 1922 et 1929, cette raison fut mentionnée explicitement. Parfois, les contrôleurs motivaient leur demande. Ainsi par exemple demandèrent-ils dans le cas de la brève comédie américaine SUSIE'S SCHEME (1917, metteur en scène inconnu, EU) d'enlever du film les images avec des fusils, car « les scènes de revolvers sont toujours nuisibles pour des enfants ». Montrer des fusils s'avéra au sein de cette catégorie effectivement la raison la plus fréquemment invoquée pour couper, suivi par le meurtre et le vol. Une impression trop foudroyante sur les enfants constituait une raison pour couper les scènes violentes ou criminelles, la crainte de l'imitation une autre. La crainte d'un comportement de reproduction de la part des jeunes n'était pas isolée. Le juge des enfants Paul Wets cité par Vandervelde au parlement, décrivit déjà en 1919 la jeunesse de l'époque. La période de l'après-guerre était d'après Wets une source de grande confusion. Les familles éclatées ou cherchant un nouvel équilibre d'après le retour ou l'absence définitive des combattants après la guerre étaient nombreuses. Les écoles fermèrent souvent leurs portes pendant un certain temps en raison de problèmes pratiques. Wets avançait que dans ces temps chaotiques de reconstruction, les enfants demeuraient seuls, ne pouvant compter que sur eux-mêmes et étaient exposés aux dangers de la rue. Il décrivait comment ces enfants, poussés par la faim et la misère croissante, écumaient les rues et volaient dans les étalages, les magasins ou les terrains en construction. Il décrivait comment ils s'emparaient de façon illégitime de jouets, de pain et d'autres produits alimentaires ${ }^{1}$.

Le début des années vingt n'apportait en outre, contrairement à ce que font supposer les chiffres du chômage et des salaires, pas d'amélioration notoire des conditions de vie, puisque le pouvoir d'achat subit une grande érosion due aux prix à la consommation croissants, l'infla-

1 P. Wets, La guerre et l'enfant, Mol, 1919, p. 10. 
tion et une forte dévaluation du franc belge en 1925. A partir de 1926, une reprise économique se fit jour, à laquelle il fut brusquement mis fin en 1929 après le crash de Wall Street. C'est dans ce contexte social qu'il faut voir le fait que le vol, même d'objets triviaux comme du beurre ou un portefeuille, était donné comme motif de la coupure de scènes dans environ dix pour cent des cas. En 1929 ce pourcentage se rapprochait même de vingt pour cent.

Une seconde grande catégorie constituant une pierre d'achoppement pour les contrôleurs des films, se rapportait à la sexualité, l'érotisme et au caractère physique. Cette catégorie occupait environ dixhuit pour cent de tous les motifs cités de coupures. Il s'agissait principalement de scènes de baisers, de personnages qui dansaient ou de scènes d'amour présentant des acteurs peu vêtus.

La dernière grande catégorie, qui se heurtait dans dix pour cent des cas au refus du contrôle des films, se rapportait au mariage et plus particulièrement au fait de montrer l'adultère ou de le rendre séduisant. Les contrôleurs considéraient de toute évidence le mariage comme la pierre angulaire de la société. L'adultère, un facteur menaçant pour la survie de la famille, ne pouvait être ni suggéré ni montré. Le mot lui-même était frappé d'interdit. Ainsi des mots tels que "amant" et "maîtresse" disparurent systématiquement des titres, ou ils étaient remplacés par d'autres mots, si bien que des amants devinrent souvent de simples amis ou même des parents plus ou moins proches. Il en fut ainsi dans le cas du film LA COURSE À L'AMOUR (1923, Paul Barlatier \& Charles Keppens, France) où la commission donna la recommandation suivante: "Remplacez le mot amant par ami". Bien qu'il ne soit pas clair à quel point les opinions catholiques courantes concernant la famille prévalaient au sein de la commission du contrôle des films, les contrôleurs faisaient preuve d'une forte préoccupation à l'égard de la réussite du mariage des jeunes filles. Au moyen de ses coupures, la commission belge de contrôle des films essayait d'éviter que les jeunes voient des filles quittant la maison paternelle contre le gré de leur père.

Outre ces grandes catégories, le suicide constituait lui aussi un tabou important. Toute référence ou représentation du suicide était systématiquement coupée. Sans cela, le film ne pouvait pas porter la mention "Enfants admis" pour être joué au cinéma, puisque les membres de la commission considéraient que le "Suicide est toujours contagieux 
pour les enfants ${ }^{1}$ ". Les contrôleurs belges des films enlevèrent toutes les références à la façon dont un personnage désire mettre fin à sa vie, ainsi qu'à ses raisons. Une des solutions pour cette menace de l'état d'esprit des enfants, c'est de faire passer le suicide pour une mort naturelle. Ainsi les contrôleurs modifièrent le film américain DARKNESS AND DAYLIGHT (1923, A. Plummer, EU) de la façon suivante: "Le scénario devrait être mis en concordance transformant le suicide en accident', et une exclamation claire telle que «Eduard s'est tué, il s'est tué ! » lorsqu'un personnage principal se suicide consécutivement à une sévère humiliation dans SIEGE (1925, S. Gade, EU), fut modifiée d'après le conseil de la commission et devint l'expression plus neutre: "Il est mort ${ }^{2} "$

\section{Critique persistante du secteur cinématographique}

La mesure temporaire pour accoutumer les distributeurs et les exploitants à la nouvelle loi ne suffit pas pour étouffer les protestations. Les premières doléances concernant tant le fonctionnement que la composition de la commission de contrôle des films se firent entendre rapidement. Dans les années vingt, la commission se composait en moyenne d'hommes à $70 \%$, souvent d'âge moyen ou plus. En outre, le poids des représentants de l'industrie cinématographique, qui constituaient généralement vingt pour cent de la commission, n'était pas suffisant pour inciter les autres membres (généralement des juristes, des instituteurs et des journalistes) à la clémence ${ }^{3}$.

Les membres de la commission pouvaient à peine compter sur la sympathie ou la confiance de l'industrie cinématographique. Encore avant l'application de la loi, Le Soir, journal important de Bruxelles, publia une lettre de La Chambre Syndicale de la Cinématographie, dans laquelle les contrôleurs étaient vivement critiqués. Le groupe de pression reprocha aux contrôleurs leur ignorance au niveau du cinéma. Le fonctionnement et surtout l'arbitraire de la commission furent par

1 Paroles prononcées dans le cadre des coupures du film VISAGES D'ENFANTS (1925, J. Feyder, France).

2 Tous les pourcentages sont calculés d'après les descriptions des coupures de tous les films présentés à la commission de contrôle belge des films entre le 1er janvier 1922 et le 31 décembre 1929.

3 S. Grénez, La commission de contrôle des films cinématographiques, (1920-1960), Bruxelles, ULB, 1989. 
conséquent souvent incriminés. Les cinéphiles et les professionnels se plaignaient en outre que les coupures dans les films portaient atteinte à la valeur artistique et à l'intégrité.

Outre le mécontentement causé par la composition de la commission et le contrôle sévère, les motifs des coupures étaient également critiqués. Cette critique provenait principalement de groupes de pression, qui demandèrent avec insistance des critères bien définis pour procéder à des coupures. D'une part il y avait les exploitants et les distributeurs de films, qui, pour citer Rens, trouvaient exagéré que "la simple exhibition d'un revolver, d'une petite femme en combinaison rend l'oeuvre indésirable pour les enfants de moins de 16 ans ${ }^{1}$." De l'autre côté, des groupes d'action catholique militèrent aussi pour un contrôle plus strict et d'inspiration plus religieuse.

Le malaise de ces groupes fut en tout état de cause à la base du fait que le fonctionnement de la commission officielle de contrôle des films fut réajusté à maintes reprises au moyen d'une longue série d'Arrêtés Royaux. En dépit de toutes ces adaptations, les fondements du contrôle belge des films furent à peine touchés au cours de son existence octogénaire.

\section{Conclusion}

La loi belge du contrôle des films de 1920, par ailleurs toujours en vigueur, est née de la crainte de la société que le film soit une école de crime et d'immoralité. Son succès croissant allait créer en Belgique, comme dans bien d'autres pays, une panique (médiatique) morale, faisant craindre l'influence néfaste du nouveau médium populaire sur les valeurs sociales essentielles. Pourtant, cela allait prendre plus de huit ans avant que cette crainte fut coulée dans une loi.

Pendant huit ans quelques problèmes importants à la croisée des chemins de la liberté d'expression, de la liberté des média, de l'ordre moral et public furent mis en discussion. Ainsi, au début du siècle précédent, la crainte de la censure était bien vivante, ainsi que la question de savoir si le pouvoir décisionnel ne revenait pas aux parents, comme la question des compétences en matière de moralité publique. Certains politiciens avançaient que le film ne tombait pas sous le coup de l'ar-

1 Voir R. Rens, Revue Belge du cinéma, 13 juin 1926, et R. Rens, Revue Belge du cinéma, 28 février 1926. 
ticle 25 de la constitution, le législateur de l'époque n'ayant jamais pu s'imaginer un tel médium nocif.

Contrairement à la plupart des pays occidentaux, le législateur belge a réussi à promulguer une loi ne pouvant être qualifiée de censure au sens strict, ce qui fait que la Belgique n'a jamais connu de censure de films pour les adultes. La solution résidait dans un contrôle facultatif, n'ayant pas lieu sous la contrainte.

La Loi Vandervelde fut de cette façon le résultat d'un difficile exercice d'équilibre entre les désirs d'intérêts très divergents dans la société de l'époque. D'une part le législateur devait tenir compte d'impératifs économiques et de dispositions constitutionnelles concernant la liberté de la presse et la liberté d'expression. D'autre part, ces intérêts devaient être jetés dans la balance contre les arguments d'éducateurs, de juges des enfants et d'autres groupes de pression qui faisaient usage d'un discours moral pour protéger la vulnérabilité d'enfants et de jeunes. La loi belge de contrôle des films essayait donc de transiger entre les idées libérales et les aspirations conservatives, souvent paternalistes.

L'introduction et la mise en route du contrôle ne se firent pas sans remous. Surtout lorsque les décisions de la commission se firent de plus en plus sévères et qu'à la fin des années vingt les coupures furent effectuées dans de nombreux films, les distributeurs et les exploitants continuèrent à militer en faveur d'une réforme en profondeur et même de l'abolition du contrôle des films. Bien que le marché belge des films se caractérisait par une grande ouverture, le contrôle des films se mua vite en censeur sévère et fut très sensible à des images présentant (à son sens) de façon trop explicite la violence, le crime, l'érotisme, l'adultère et le suicide. 